\title{
Percepciones sobre la tutoría entre pares en escritura académica
}

\author{
Escrito por Pilar Mirely Chois-Lenis \\ Universidad del CAUCA \\ Popayán, Colombia \\ pilarchois@unicauca.edu.co \\ Adriana Carolina Casas-Bustillo \\ UnIVERSIDAD DEL CAUCA \\ Popayán, Colombia \\ acasas@unicauca.edu.co
}

\section{Resumen}

Esta investigación cualitativa retomó elementos de la etnografía educativa y de la fenomenología, para comprender las percepciones de los participantes en tutorías de escritura académica entre pares. Los hallazgos permitieron advertir que tutor y tutorado perciben beneficios tanto en el campo académico como en el social; cada uno valora positivamente en el otro algunos rasgos de personalidad, y también otros atribuibles específicamente a sus roles de aprendiz y tutor. Se concluye que la tutoría entre pares, asumida como una relación parcialmente asimétrica, puede ser una estrategia valiosa en los centros de escritura para favorecer el aprendizaje de la producción textual, si se cuenta con condiciones como la formación permanente de tutores.

\section{Palabras clave}

Percepción; tutoría; escritura; etnografía
AMPARO LÓPEZ-Higuera

Universidad del Cauca Popayán, Colombia alopezh@unicauca.edu.co

Diana Marcela Prado-Mosquera Universidad del CAUCA Popayán, Colombia dmprado@unicauca.edu.co

Edwin Yamir Cajas-Paz Universidad del Cauca Popayán, Colombia edwincajasp@gmail.com

Para citar este artículo / To cite this article / Pour citer cet article / Para citar este artigo

Chois-Lenis, Pilar Mirely; Casas-Bustillo, Adriana Carolina; López-Higuera, Amparo; Prado-Mosquera, Diana Marcela \& Cajas-Paz, Edwin Yamir (2017). Percepciones sobre la tutoría entre pares en escritura académica. magis, Revista Internacional de Investigación en Educación, 9 (19), 165 184. doi: 10.11144/Javeriana.m9-19.ptpe

\section{Transferencia a la práctica}

Los hallazgos de esta investigación podrían motivar la implementación de tutorías de escritura académica entre pares en programas y centros de escritura que pretendan contribuir a la formación escritural de estudiantes universitarios. Ofrece pistas sobre criterios a considerar en la selección y formación de tutores, pues evidencia que el solo hecho de ser par no garantiza un desempeño tutorial adecuado. También permite identificar algunas prácticas tutoriales que los participantes perciben como positivas, como la capacidad de promover la reflexión sobre el proceso de producción textual por parte del tutor y, en consecuencia, la ganancia progresiva de autonomía en esta tarea por parte del tutorado. 


\section{Keywords}

Perception; tutorial;

writing; ethnography

\section{Mots clés}

Perception; tutorat;

écriture; ethnographie

\section{Abstract}

This qualitative research included elements of educational ethnography and phenomenology to understand participants' perceptions of peer academic writing tutorials. Evidence suggest that both, tutor and mentor, perceive academic and social benefits; each one positively values in the other some personality traits, as well as other characteristics specifically related to the roles of apprentice and tutor. It is concluded that peer tutoring, understood as a partially asymmetrical relationship, can be a valuable strategy in writing centers that could favor the learning of textual production if some conditions are considered, such as the ongoing education of tutors.

\section{Transfer to practice}

Results could encourage the implementation of academic peer writing tutorials in programs and writing centers in order to contribute to the development of writing skills among university students. This paper offers ideas about criteria that need to be considered in the selection and education of tutors; it evidences that the mere fact of being a peer does not guarantee an adequate tutorial performance. This paper also helps to identify some tutorial practices that participants perceive as positive, such as the ability to promote reflection on the process of textual production by the tutor and, consequently, the progressive autonomy developed in this task by the student.

\section{Résumé}

Cette recherche qualitative reprend les éléments de l'ethnographie éducative et de la phénoménologie, pour comprendre les perceptions des participants dans les tutorats d'écriture académique entre pairs. Les trouvailles nous ont permis de voir que le tuteur et le tutoré reçoivent des rétributions tantôt vis-à-vis du domaine académique qu'au niveau social; chacun apprécie positivement dans son pair quelques traits de personnalité, et aussi d'autres traits qui sont attribués spécifiquement à leurs rôles de débutant et tuteur. Donc le tutorat entre pairs, qui est pris en tant que rapport partiellement asymétrique, peut être une stratégie puissante dans les centres d'écriture pour favorise l'apprentissage de la production textuelle; si I'on compte avec les conditions telle que la formation permanente de tuteurs.

\section{Transfert à la pratique}

Les trouvailles de cette recherche peuvent encourager l'implémentation de tutorats d'écriture académique entre pairs dans les programmes et centres d'écriture qui visent contribuer à la formation de leurs étudiants universitaires dans la production écrite. On donne des pistes par rapport aux critères à prendre en compte dans le choix et la formation de tuteurs, car on montre que le seul fait d'être pair n'est pas suffisant pour assurer le bon exercice de tutorat souhaité. Par ailleurs, on a identifié quelques pratiques de tutorat que les participants ont reconnu comme positives, par exemple la capacité d'encourager la réflexion par rapport au processus de production textuelle de la part du tuteur et, en conséquence, de l'avantage progressif d'autonomie dans cette tâche de la part du tutoré.

\section{Palavras-chave}

Percepção; tutoria;

escrita; etnografia

\section{Resumo}

Esta investigação qualitativa retomou elementos da etnografia educativa e da fenomenologia, para compreender as percepções dos participantes em tutorias de escrita acadêmica entre pares. Os resultados permitiram advertir que tutor e tutorado percebem benefícios tanto no campo acadêmico como no social; cada um valoriza positivamente no outro alguns traços de personalidade, e também outros atribuíveis especificamente a seus papéis de aprendiz e tutor. Conclui-se que a tutoria entre pares, assumida como uma relação parcialmente assimétrica, pode ser uma estratégia valiosa nos centros de escrita para favorecer a aprendizagem da produção textual, se se conta com condições como a formação permanente de tutores.

\section{Transferência à prática}

Os resultados desta pesquisa poderiam motivar a implementação de tutorias de escrita acadêmica entre pares em programas e centros de escrita que pretendam contribuir à formação de estudantes universitários. Oferece pistas sobre critérios a considerar na seleção e formação de tutores, pois evidencia que o simples fato de ser par não garante um desempenho tutorial adequado. Também permite identificar algumas práticas tutoriais que os participantes percebem como positivas, como a capacidade de promover a reflexão sobre o processo de produção textual por parte do tutor e, em consequência, o ganho progressivo de autonomia nesta tarefa por parte do tutorado. 


\section{Introducción}

La tutoría entre pares es concebida como un método de aprendizaje cooperativo basado en la organización de parejas: tutor y tutorado (Grupo de investigación sobre aprendizaje entre iguales de la Universidad Autónoma de Barcelona [GRAI], 2007; Moliner-Miravet, Sales-Ciges \& MolinerGarcía, 2014). Por lo general, el aprendizaje cooperativo se comprende como un proceso caracterizado por la participación activa, responsable, intencional y articulada de cada uno de los miembros de un grupo, quienes pretenden contribuir al desarrollo de habilidades de los demás y de sí mismos (Domingo, 2008; Johnson, Johnson \& Holubec, 1999); además de perseguir una meta común o el desarrollo de un producto final (Panitz, 1999). Esta caracterización del aprendizaje cooperativo permite señalar que, si bien se espera que la tutoría entre pares demande la participación activa de tutor y tutorado, estos actores no persiguen un objetivo común, pues se encuentran en una relación asimétrica, dada centralmente por la intencionalidad del primero de apoyar el aprendizaje del segundo.

Esto no significa que se desconozca la posibilidad de aprendizaje del tutor pues tal como señala Keith J. Topping (1996), la tutoría entre pares implica que personas de grupos sociales similares, sin ser profesores profesionales, se ayuden entre sí a aprender, y aprendan ellas mismas enseñando. Para este autor, no se trata necesariamente de la transmisión por parte de alguien más capaz y experimentado — quien ya ha desarrollado ciertos conocimientos y habilidades - a alguien menos apto - quien todavía tiene que desarrollarlos-.

Sin embargo, para Janet W. Colvin y Marinda Ashman (2010) tanto en la tutoría como en la mentoría entre pares, quien orienta y quien es orientado no están estrictamente en las mismas condiciones. En el primer caso, se trata de un estudiante más aventajado que ayuda a otro de nivel inferior para contribuir a la apropiación de los contenidos de un curso en particular; mientras que en el segundo caso se trata de un estudiante más experimentado que ayuda a otro menos experimentado a mejorar su rendimiento académico general y su crecimiento personal.

Desde esta perspectiva de la tutoría entre pares como una relación asimétrica, la Asociación Nacional de Universidades e Instituciones de Educación Superior de México (ANUIES, 2000) considera al tutor como un guía en el proceso formativo del estudiante, que conscientemente lo orienta, asesora y acompaña, hacia su formación integral. En el mismo sentido, Lina Marcela Duque-Ossa, Vittoria Gómez-Martínez y Ana Sofía Gaviria-Cano (2009) refieren que quien cumpla ese rol debe implementar estrategias que permitan la integración de conocimientos y promuevan la autonomía del estudiante. El tutorado, por su parte, es considerado por Luis Enrique Jiménez-Becerril (2009) como una persona "novata, aprendiz, menos experta y protegida", que debería tener, según Howard G. Adams (1993), responsabilidad, iniciativa, ingeniosidad y habilidad para lograr sus metas, tomando en cuenta los consejos dados por el tutor.

Otras investigaciones han revelado que la tutoría entre pares puede generar diversos beneficios. Por ejemplo, políticamente, permite delegar la gestión del aprendizaje a los estudiantes de manera democrática con miras a empoderarlos (Topping, 1996); además contribuye al aprendizaje profundo, la cooperación y los logros personales de los participantes (Loke \& Chow, 2007).

Específicamente, los tutores pueden recibir beneficios como el incremento de sus niveles de productividad (Lucas, 2000), satisfacción personal, aumento de la autoestima (Lucas, 2000; Moliner-Miravet, Sales-Ciges \&
Descripción del artículo | Article description | Description de l'article | Artigo descrição

Este artículo de investigación presenta algunos resultados del proyecto de investigación Tutoría de escritura entre pares: prácticas y percepciones, registrado en la Vicerrectoría de Investigaciones de la Universidad del Cauca; ID 4077, cuya investigadora principal es la profesora Pilar Chois. 
Moliner-García, 2014), y la transformación e incremento de los conocimientos y habilidades que tienen lugar gracias al trabajo que implica el proceso de enseñanza (Lyons, Scroggins \& Rule, 1990; Sharif, Zakaria, Wan Mansor, Nordin, Ng \& Mustafa, 2012; Topping, 1996). Se destaca, por ejemplo, que actividades que realiza el tutor, como la explicación y la interrogación, potencian el crecimiento de su rol (Galbraith \& Winterbottom, 2011; Roscoe \& Chi 2008). Más allá de los aportes en lo académico, Jolan T. Nisbet, Mark D. Haw y Ashleigh J. Fletcher (2014) reconocen que los tutores se benefician en aspectos laborales como el desarrollo de sus hojas de vida y la solvencia en entrevistas de trabajo.

Los tutorados, por su parte, no temen admitir sus inquietudes y plantear preguntas gracias al clima de confianza que establecen con sus pares, sin la presión propia de la interacción maestro-estudiante (Moliner-Miravet, Sales-Ciges \& Moliner-García, 2014). También se destaca la mejora de su experiencia académica, gracias a que obtienen nuevos conocimientos (Lucas, 2000), al involucrarse de manera más activa, interactiva y participativa en su aprendizaje (Topping, 1996) y desplegar la capacidad de identificar los recursos personales que les permiten desarrollar sus propias habilidades (Santiviago \& Mosca, 2012). Además de las ganancias cognitivas inmediatas, se reconoce una mayor conciencia metacognitiva y una mejor aplicación de conocimientos y habilidades a nuevas situaciones (Topping, 1996); así como también ganancias personales —incremento de autoestima- y sociales —empatía con los demás e integración a la institución- (Santiviago \& Mosca, 2012; Topping, 1996).

A pesar de los aportes de esta modalidad de enseñanza y aprendizaje, también se pueden identificar en ella retos y dificultades. Por ejemplo, Lisa C. Ehrich, Brian C. Hansford y Lee Tennent (2003) mencionan que generalmente se presenta poca disponibilidad en cuanto al tiempo por parte del tutor para atender sus deberes tutoriales, además de problemas interpersonales, incompatibilidad de caracteres, falta de compromiso y distancia entre las expectativas del tutor y el tutorado. Jeffrey Lee Lucas (2000), por su parte, manifiesta que las relaciones entre los participantes pueden resultar explosivas o discriminativas, y que se puede generar dependencia o disminución del potencial del alumno que asiste a la tutoría. Así mismo, los tutores afirman que la pasividad en el aprendizaje, el poco compromiso y la impuntualidad de los tutorados afectan el desarrollo de su labor; además, les preocupa sobre sí mismos no tener suficiente conocimiento para enseñar y la existencia de estilos de aprendizaje y rasgos de personalidad no coincidentes entre ambos participantes (Loke \& Chow, 2007).

Específicamente en el campo de la escritura académica, es usual hablar de la revisión entre pares como una práctica de evaluación de diferentes tipos de discurso, en la que un estudiante valora críticamente un texto escrito por otro. Entre los beneficios de la revisión de textos entre pares, de acuerdo con Paula Carlino (2008b), se puede incluir que permite a los autores aprender a tomar las sugerencias de los lectores, no como algo que deba hacerse para cumplir una tarea, sino como algo que permite examinar y evaluar su desempeño durante la escritura, pues el estudiante puede decidir sobre su propio plan de mejora como autor del texto.

Este modo de cooperación no solo genera beneficios para la elaboración de los escritos, también activa procesos metacognitivos relacionados con la revisión de los mismos (Cassany, 1999). Así mismo, las conversaciones y lecturas compartidas entre estudiantes les permiten participar en un proceso de comunicación en torno a la escritura, tomar conciencia sobre sus propias dificultades, encontrar posibles soluciones ante los errores y resolverlos de forma cooperativa (Alzate-Medina \& Peña-Borrero, 2009). 
Sin embargo, los riesgos no están ausentes en esta práctica. Por ejemplo, de acuerdo con Alfonso Vargas-Franco (2014), pueden darse algunos problemas concernientes a la falta de conocimiento del estudiante para corregir los textos de sus compañeros en todos los aspectos y a la desconfianza por parte de su par por el trabajo que el primero realiza, razón por la que algunos alumnos consideran esta modalidad como una pérdida de tiempo y prefieren la revisión de su escrito por parte de un docente.

Ahora bien, es conveniente distinguir entre revisión entre pares — también llamada revisión colaborativa- y tutoría entre pares en el campo de la escritura académica. Ambas se pueden definir de manera amplia como una conversación en torno a un texto, basada en la valoración que de él hace principalmente uno de los participantes, con miras a la cualificación del documento y al aprendizaje de la escritura. No obstante, la revisión entre pares no implica obligatoriamente que quien hace la valoración del documento cuente con la formación necesaria para comprender el proceso de escritura, considerar los criterios pertinentes durante la evaluación de un texto o para orientar a un par en la revisión y reescritura de su documento. La tutoría, por su parte, sí implica que un estudiante más aventajado oriente el proceso, con base en una formación que le permite la toma razonada de decisiones.

De acuerdo con Alfonso Vargas-Franco (2014), la revisión entre iguales se da durante el proceso de producción escrita planificado en el marco de una secuencia de aprendizaje; ese proceso incluye la escritura de borradores, "los cuales son intercambiados, leídos y comentados por un compañero antes de la revisión final del profesor" (p. 15). Se trata entonces, para este autor, de una práctica, dispositivo o actividad didáctica que tiene lugar durante un curso. Puede asumirse entonces que la secuencia de aprendizaje incluye momentos en que los estudiantes apropian criterios para la valoración textual y su comunicación, o que han construido previamente tales saberes, pero se trata de una relación simétrica y con roles intercambiables. Así se ha documentado en trabajos de pregrado: Mariona Corcelles-Seuba, Maribel Cano-Ortiz, Gerardo Bañales-Faz y Norma Alicia Vega (2013); Karen Shirley López (2012); Marta Pardo y Montserrat Castelló (2016) y Alfonso Vargas-Franco (2014); Anne Venables y Raymond Summit (2003), y de posgrado: Paula Carlino (2008a, 2009); Montserrat Castelló, Dolores González y Ana Iñesta (2010); Olga Dysthe y Sølve Lillejord (2012); Rudy Mostacero (2014); Cecilia Pereira y Mariana di Stefano (2007).

Al contrario, la tutoría entre pares o iguales, según Gloria Mercedes Alzate-Medina y Luis Bernardo Peña-Borrero (2010), está basada en una relación asimétrica entre el tutor y el tutorado, por cuanto se reconoce un mayor nivel de competencia en el primero, lo cual no invalida la potencialidad del aprendizaje mutuo basado en la interacción. La condición de pares está dada por el rol de estudiantes de ambos participantes, lo cual puede aminorar la distancia que caracteriza las relaciones entre docente y alumno, y permitir un clima más tranquilo y de confianza.

Asuntos como: a. el tutor no está obligado a asignar una nota, b. no se trata de un espacio regulado por el docente que revisará el trabajo, c. ambos participantes tienen edades similares y desempeñan el rol de estudiantes universitarios y $d$. han vivido experiencias de escritura similares en tiempos cercanos (Moust \& Schmidt, 1994), marcan una diferencia notable con respecto a la relación entre profesor y alumno. Se trata, entonces, de una relación no tan vertical y asimétrica como la usual entre profesor y estudiante, pero tampoco tan horizontal como la que se deriva del desconocimiento de diferentes niveles de competencia en el campo de la escritura académica de los participantes.

En consideración a las potencialidades de la tutoría entre pares, pero también anticipando sus posibles riesgos, los gestores del Centro de Escritura de la Universidad del Cauca asumieron esta modalidad de intervención desde su creación en 2013. Esto implicó la construcción de una infraestructura de trabajo que incluía un proceso de formación permanente de tutores. Las valoraciones hechas por los tutorados que asistieron al Centro durante el primer año de funcionamiento fueron predominantemente positivas, pero el interés por indagar a fondo tanto lo que sucedía en este espacio como la percepción de sus protagonistas al respecto motivó el desarrollo de una investigación que tuvo como objetivo general comprender las prácticas y percepciones que se construyen en el marco de las tutorías en escritura académica entre pares. Para ello, se plantearon tres objetivos específicos: a. conocer las experiencias de los participantes en cuanto al rol de tutor y tutorado, b. reconocer sus interpretaciones frente a las situaciones presentes en el desarrollo de las tutorías y c. identificar los juicios planteados respecto a las tutorías desarrolladas en el Centro de Escritura.

En este artículo, se presentan los resultados que corresponden a las percepciones que, sobre las tutorías entre pares, han construido tutores y tutorados. La percepción es definida por Maurice Merleau-Ponty (1975) como un fenómeno que no solo comprende procesos fisiológicos, sino que implica la búsqueda de significados de lo que, según refieren Luis Guillermo Jaramillo-Echeverri y Juan Carlos Aguirre-García (2012), es el trasfondo de los actos de la realidad en el campo fenoménico del hombre. Toda percepción, de acuerdo con Merleau-Ponty (1975), empieza con la sensación como forma de aproximarse a la realidad; 
esta aproximación recoge información que se reestructura gracias a pasos o etapas de selección, organización e interpretación que le permiten al individuo proporcionar significación a los estímulos percibidos y, a partir de esto, formular un juicio sobre ellos (Guardiola-Jiménez, 2013).

Las percepciones, entonces, pueden constituir un motor de acción, pues se actúa de acuerdo con la significación que se atribuye a una situación en particular. En ese sentido, dar cuenta de las percepciones de los participantes en las tutorías de escritura académica entre pares puede, además de aportar conocimiento sobre esa modalidad de trabajo académico, servir de base para realizar acciones tendientes a cualificar las prácticas que se desarrollan en este espacio y en otros que persigan propósitos semejantes.

\section{Metodología}

El Centro de Escritura de la Universidad del Cauca, contexto donde se desarrolló la presente investigación, fue creado por el programa académico de Fonoaudiología en el primer período de 2013, con el objetivo de potenciar las habilidades de escritura académica en la comunidad universitaria. Asisten desde entonces todos los estudiantes, profesores, directivos o administrativos que deseen cualificar su desempeño en escritura académica o recibir apoyo en la escritura de un texto en particular. Allí se desarrollan talleres grupales sobre las características de un tipo de texto determinado, su procedimiento de producción, u otros asuntos relacionados de acuerdo con las demandas particulares de los interesados.

Los profesores también pueden obtener orientación sobre maneras de potenciar el uso de la lectura y la escritura, como herramientas de aprendizaje, en las clases que tienen a cargo. El servicio más solicitado son las tutorías personalizadas de escritura, dirigidas a personas que requieren apoyo en cualquiera de las fases del proceso de realización de un texto: en la planificación, la escritura de borradores o la revisión y reescritura hasta lograr la versión definitiva del escrito.

Esas tutorías las desarrollan estudiantes de séptimo y décimo semestre del Programa de Fonoaudiología, quienes fueron seleccionados por sus habilidades escriturales y buenas relaciones interpersonales. Ellos participaron, antes de fungir como tutores, en un proceso de capacitación orientado a la apropiación de herramientas conceptuales y metodológicas sobre la escritura académica. Además de la capacitación inicial, el grupo de tutores participa periódicamente en espacios de reflexión teórico práctica y cuenta con la orientación permanente de la coordinadora del Centro.

El desarrollo de esta investigación se inscribió en la etnografía particularista propuesta por Joyceen S. Boyle (2003), pues centró la atención en un grupo localizado de personas que comparten características culturales y sociales (estudiantes), están concentradas en un sitio (un centro de escritura) y desarrollan una actividad específica (tutoría entre pares). La etnografía educativa aportó fundamentos metodológicos para comprender actitudes, opiniones y creencias de un fenómeno educativo desde las perspectivas de los estudiantes (Govea-Rodríguez, Vera \& Vargas, 2011). Además, retomando la referencia a Maurice Merleau-Ponty (1975), la percepción aunque no pura ni definitiva - sirve de acceso al campo fenomenal de la realidad, siendo los juicios la forma de aproximarse a ella; como expresión singular que puede llegar a convertirse en un sentido universal de lo que se pretende comprender (Josgrilberg, 2008).

Los informantes se seleccionaron de manera no probabilística teniendo en cuenta al tutor y al tutorado que participaron en una misma tutoría. 
En el caso de los tutorados, fueron estudiantes de pregrado que asistieron al Centro de Escritura de la Universidad del Cauca y los tutores, quienes se desempeñaron en este rol durante el primer período académico de 2014: 6 estudiantes del programa de Fonoaudiología de séptimo semestre y 3 de décimo semestre. En ambos casos, se contó con el consentimiento informado, cuya firma demostraba la participación voluntaria en el estudio; además era necesario que tutores y tutorados fuesen estudiantes, a fin de asegurar su condición de pares. Para comprender las prácticas y las percepciones en torno a la tutoría entre pares, se usaron técnicas como la observación de las sesiones de tutoría, que fueron registradas en audio y posteriormente transcritas usando convenciones para dar cuenta de aspectos tonales y pausas significativas. Además, se elaboró y administró una entrevista estructurada abierta en la que, por definición, las preguntas se redactan y ordenan con antelación (Vargas-Jiménez, 2012), pero permite a los entrevistadores promover en el entrevistado hablar sin contradecirlo, sin sesgarlo, y realizar contrapreguntas en caso de que las respuestas no sean consideradas suficientes o el entrevistado solicite aclaración de la pregunta (Valles, 1999).

Cada entrevista fue grabada y transcrita de acuerdo con el sistema de convenciones retomado de Carsten Sinner (2001), para dar cuenta exacta de lo dicho por los participantes y marcar algunos aspectos lingüísticos y no lingüísticos de interés, como muestra en la tabla 1.

Tabla 1

Sistema de convenciones usado para la transcripción de entrevistas

y grupo de discusión

\begin{tabular}{l|l}
\hline Subrayado & Acentuación \\
\hline$i ?$ & Interrogación \\
\hline i! & Exclamación \\
\hline$/$ o / & Interrupciones \\
\hline$($, coma $)$ & Pausa corta \\
\hline$<\ldots>$ & Pausas largas \\
\hline$<$ riendo $>$; $<$ apunta el libro $>$ & $\begin{array}{l}\text { Indica aspectos paralingüísticos, cinéticos, } \\
\text { extralingüísticos de interés. }\end{array}$ \\
\hline$<$ ininteligible $>;$ & Partes de las entrevistas incomprensibles \\
\hline$<?>$ palabra $<?>$ & Partes solo inteligibles por el contexto \\
\hline \hline
\end{tabular}

Fuente: elaboración propia con base en la propuesta de Carsten Sinner (2001)

En total, se obtuvieron 80 entrevistas, de las cuales 40 se realizaron a igual número de tutorados, mientras que las cuarenta restantes fueron realizadas a 9 tutores que respondieron con base en las tutorías en las que acababan de participar. Las entrevistas se tomaron en el transcurso de un período académico y se realizaron una vez finalizada la tutoría sobre la cual se indagaba. Para el caso de los tutores, algunas preguntas del instrumento que indagaban sobre su sentir respecto a ser tutor y los beneficios logrados en este rol, se hicieron solo una vez durante el trabajo de campo.

También se desarrolló un grupo de discusión con varios tutorados, mediante el cual se indagaron algunos asuntos que interesaron a los investigadores después de un primer análisis de las entrevistas, como el impacto de las tutorías sobre el aprendizaje del tema abordado en el texto objeto de revisión. 
En este artículo se presentan específicamente los resultados correspondientes a la indagación de las percepciones mediante la entrevista y el grupo de discusión. Considerando el flujo de información transcrito y el análisis línea a línea, se eligieron el método analítico propuesto por Barney G. Glaser y Anselm L. Strauss (1967) y los planteamientos de Miguel S. Valles (1999). Esta forma de aproximarse a los registros permitió el acercamiento a puntos de encuentro en los datos y dio forma a una codificación inicial en la que se asignó una denominación común a grupos de ideas, y usó como elemento organizador las preguntas formuladas en la entrevista. En segundo lugar, se realizó un proceso de codificación abierta que consistió en la búsqueda de propiedades comunes entre las denominaciones logradas en el anterior paso analítico y se asignó así una nueva categorización. A continuación, se integraron las categorías y sus propiedades como respuestas provisionales dentro del análisis, lo que permitió delimitar la teoría por medio de una serie de operaciones analíticas de codificación axial, esto es, grupos organizados de hallazgos que se aproximan a la comprensión del fenómeno de estudio, para finalmente lograr categorías conceptuales (Valles, 1999).

\section{Hallazgos}

Como parte del análisis línea a línea en la codificación, la emergencia de categorías dio lugar a elaboraciones sobre la percepción de la tutoría entre pares, como muestra la tabla 2. Los hallazgos se presentarán considerando dichas categorías.

Tabla 2

Categorías de análisis

\begin{tabular}{|c|c|c|c|}
\hline \multicolumn{4}{|c|}{ Emergencia de categorías } \\
\hline $\begin{array}{l}\text { Categorías desde la } \\
\text { codificación inicial }\end{array}$ & $\begin{array}{l}\text { Categorías desde la } \\
\text { codificación abierta }\end{array}$ & $\begin{array}{l}\text { Categorías desde la } \\
\text { codificación axial }\end{array}$ & $\begin{array}{l}\text { Categoría } \\
\text { conceptual }\end{array}$ \\
\hline \multirow{3}{*}{$\begin{array}{l}\text { 1. Percepciones sobre las } \\
\text { motivaciones para asistir a tutoría }\end{array}$} & Por exigencia docente & \multirow{3}{*}{$\begin{array}{l}\text { Intención central: terminar } \\
\text { la tutoría con una mejor } \\
\text { versión del texto }\end{array}$} & \multirow{13}{*}{$\begin{array}{l}\text { La percepción de la } \\
\text { tutoría entre pares } \\
\text { como un espacio que } \\
\text { otorga beneficios a sus } \\
\text { participantes }\end{array}$} \\
\hline & Por recomendación & & \\
\hline & Por iniciativa propia & & \\
\hline \multirow{4}{*}{$\begin{array}{l}\text { 2. Percepciones sobre los aportes } \\
\text { de las tutorías en el ámbito } \\
\text { académico }\end{array}$} & $\begin{array}{l}\text { Aportes percibidos en } \\
\text { escritura académica }\end{array}$ & \multirow{4}{*}{$\begin{array}{l}\text { Aprendizaje: la tutoría } \\
\text { no solo tiene beneficios } \\
\text { inmediatos, se extienden a } \\
\text { futuras tareas de escritura }\end{array}$} & \\
\hline & $\begin{array}{l}\text { Aportes percibidos } \\
\text { en la lectura }\end{array}$ & & \\
\hline & $\begin{array}{l}\text { Aportes percibidos en la } \\
\text { dinámica de trabajo en grupo }\end{array}$ & & \\
\hline & $\begin{array}{l}\text { Aportes percibidos en el } \\
\text { aprendizaje del tema }\end{array}$ & & \\
\hline \multirow[t]{2}{*}{$\begin{array}{l}\text { 3. Percepciones sobre los aportes } \\
\text { de las tutorías en el ámbito social }\end{array}$} & $\begin{array}{l}\text { Establecimiento de nuevos } \\
\text { vínculos de amistad }\end{array}$ & \multirow{6}{*}{$\begin{array}{l}\text { Implicaciones de la relación } \\
\text { entre pares }\end{array}$} & \\
\hline & $\begin{array}{l}\text { Toma de confianza para } \\
\text { ejercer el rol de tutor }\end{array}$ & & \\
\hline \multirow{2}{*}{$\begin{array}{l}\text { 4. Percepciones sobre las } \\
\text { características de los participantes }\end{array}$} & Sobre el tutor & & \\
\hline & Sobre el tutorado & & \\
\hline \multirow[t]{2}{*}{$\begin{array}{l}\text { 5. Percepciones sobre la relación } \\
\text { entre pares }\end{array}$} & $\begin{array}{l}\text { Una relación en la que no hay } \\
\text { posiciones de autoridad }\end{array}$ & & \\
\hline & El tutor competente & & \\
\hline
\end{tabular}

Fuente: elaboración propia 
1. Percepciones sobre las motivaciones para asistir a tutoría

Los informantes - tutores y tutorados - reconocen que los segundos asisten a tutorías con pares en el Centro de Escritura por tres tipos de motivaciones: por obligación, por recomendación y por iniciativa propia. En cuanto al primer motivo, los participantes señalan que algunos docentes exigen la revisión de sus trabajos escritos en el Centro antes de ser entregados: (Tt/04/04/14N6): "[...] nos exigen pasar por el Centro de Escritura', (T/25/03/14N3): "ellos vinieron, eeeh..., por solicitud, bueno, más que solicitud porque la profesora [...] les dijo que era, eeeh..., un requisito para la entrega del informe [...]".

Otra de las motivaciones proviene de la recomendación que docentes y amigos hicieron sobre la tutoría, sin carácter de obligatoriedad (Tt/28/04/14N14) "por recomendación de las asesoras del proyecto" y (T/08/04/14N8) "él asistió porque, porque unos compañeros de Derecho de él le recomendaron que viniera a hacer a la revisión de un memorial [...]". En el caso de la recomendación de amigos o compañeros, juega un papel importante la experiencia previa de asistencia al Centro, pues los beneficios percibidos hacen pensar que la tutoría podría también ayudar a otros.
Registros como T/28/04/14N17: "[asistió] porque tenía dificultades en la redacción de algunos párrafos del informe" o Tt/09/04/14N10: "[...] es que presentaba demasiados problemas en tanto escritura, redacción en lo que, o sea, en lo que me colaboraron acá", dan cuenta de que algunos tutorados asisten por iniciativa propia, bien sea porque reconocen las dificultades en su producción textual, o por el interés de formarse como tutores, asunto que sucede solo en el caso de estudiantes de Fonoaudiología previamente notificados sobre su posible selección como tutores.

Es evidente que una intención central de los tutorados es terminar la tutoría con una mejor versión del texto que están escribiendo, pero reconocen que obtienen ganancias que van mucho más allá de ese interés inicial, como se observó en los hallazgos sobre lo que se percibe como beneficio de las tutorías, asunto que se describirá a continuación.

\section{Percepciones sobre los aportes de las tutorías}

El gráfico 1 presenta de manera esquemática los beneficios reportados por los participantes en el estudio.

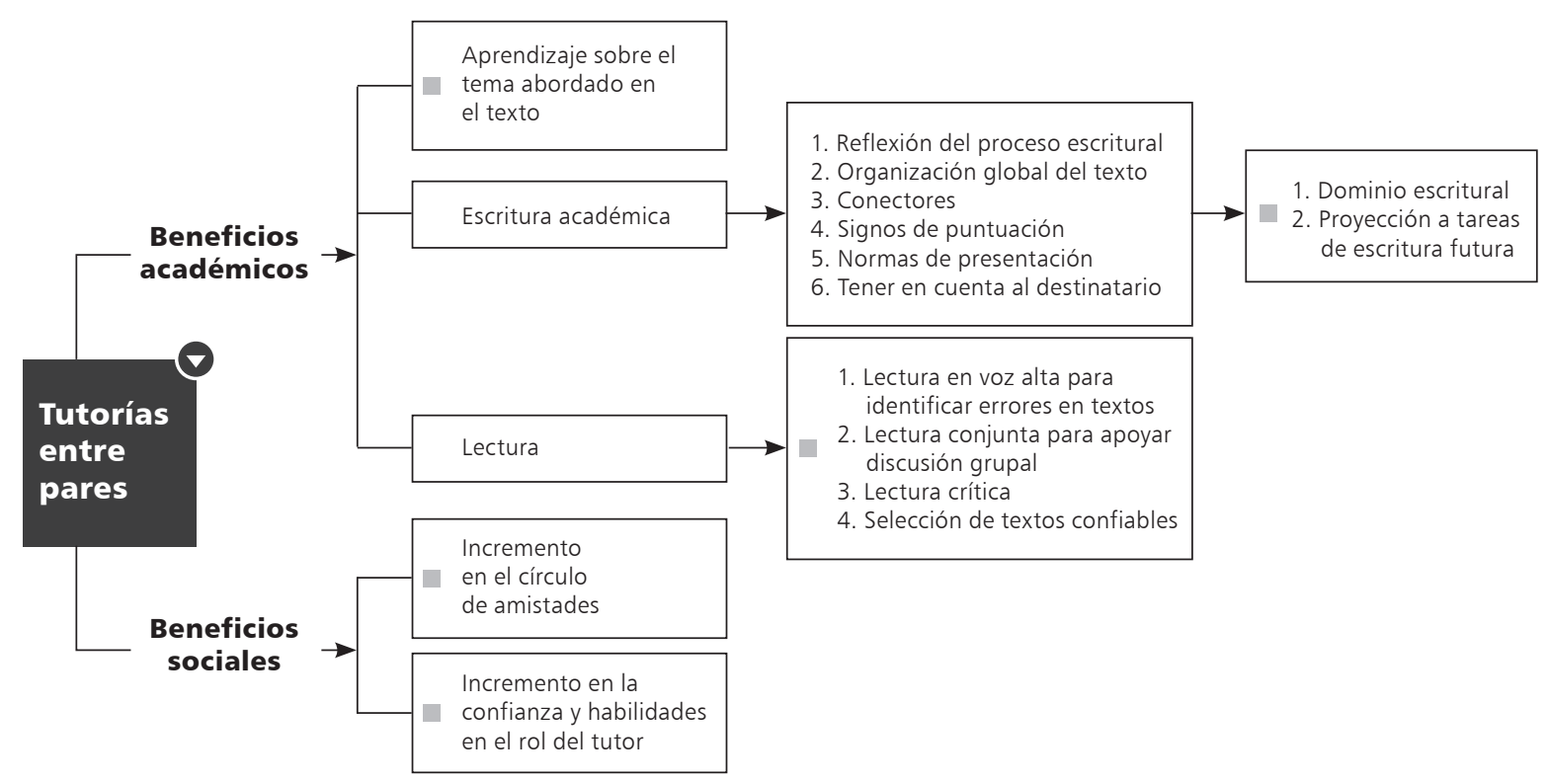

Fuente: elaboración propia

1 Los registros son transcripciones exactas de los aportes de los participantes durante las entrevistas o durante el grupo de discusión. Magis no edita las transcripciones de las entrevistas. 
Percepciones sobre los beneficios académicos

Tutores y tutorados identifican que el desarrollo de las tutorías les genera beneficios en el ámbito académico, que incluyen aportes específicos a su escritura académica. Algunos expresan que la tutoría les permitió tomar conciencia de su proceso de producción textual, reflexionar sobre él y valorarlo (Tt6/05/14N1) "para reflexionar de mi forma de escribir porque yo pensaba que escribía bien hasta que me di cuenta que [sic] había muchos errores...". Además, se encontró que preguntas que surgen regularmente durante la conversación a lo largo de la tutoría, aparecen luego para autorregular el proceso independiente de composición escrita del tutorado: (Nota del grupo de discusión) "....entonces el hecho de que siempre te digan ¿de dónde sacaste esto? o ¿esto es un planteamiento?, ¿esto es una afirmación?, tienes que referenciar..., lo lleva a uno a pensar ¿esto es verdad?, ¿yo de dónde saqué eso? [...] Entonces me parece que en las tutorías sí debe uno pensar, reflexionar y ciertamente en el ejercicio de hacer eso varias veces con el tutor, uno ya sabe, cuando está escribiendo, dice: pero tengo que tener presente que esto no lo debo colocar si no es algo verídico o si yo mismo comprobé que esto es así...".

Tutores y tutorados identifican una amplia variedad de aspectos relacionados con los textos que fueron abordados durante la tutoría y se convirtieron en objeto de aprendizaje, entre los que se resaltan la mejora en la organización global de sus textos y la coherencia: (T/25/04/14N11) “[...] nos está ayudando en la elaboración ahorita del proyecto de investigación que sabemos pues cuáles son los apartados, cómo serían como, eeeh..., la superestructura de estos textos [...]" y (T/08/04/14N8) "[...] pudimos avanzar en cuanto [...] aaah..., digamos, a que el texto estuviera bien redactado, bien escrito, estuviera coherente [...]".

Otro aporte frente a la escritura que reconocen los participantes es que las tutorías permiten al tutorado cualificar su escritura en lo que se refiere al uso de conectores y signos de puntuación, la conjugación verbal, ortografía y articulación de ideas, tal como se observa en los siguientes registros: (Tt/28/04/14N1) "pues, qué le digo, también lo de los verbos, que estén eeeh... bien conjugados, que tenga una buena articulación, una buena cohesión, las comas, los puntos y todo eso" y (Tt/20/05/14N36) "eeeh..., pues como que uno escribe las ideas pero como que no las pone muy claramente, pero entonces uno tiene que, a partir de lo que ellas nos enseñan, pues colocar los conectores y eso como para que quede el texto como más estructurado".

Otra ganancia percibida por tutores y tutorados es la capacidad de referenciar autores de acuerdo con normas y lineamientos determinados para presentar trabajos escritos: (Tt/20/05/14N34) "eeeh... cómo citar, eeeh... cuando es una cita, un autor, una página en línea [...] eeeh... márgenes según Icontec $<\ldots>$ ya". También se perciben aportes en asuntos más elaborados; por ejemplo, en relación con el propósito de la escritura y específicamente cuando se piensa en el destinatario de un texto como factor que condiciona la escritura: (Nota del grupo de discusión) "siempre manejaron como el mismo los mismos objetivos tratar de que nosotros aprendiéramos cómo ehh hacer para que el texto siempre tuviera una idea coherente en una secuencia ehh como agradable para el lector, para que él pudiera manejar el tema, entender el tema sin necesidad de mucho, ehh... sin necesidad de tener que recurrir a muchos lugares para entender lo que nosotros queríamos decir".

Resultó interesante encontrar que, en la medida en que se desarrollan varias sesiones con un mismo tutorado, se va evidenciando en este un mayor dominio escritural: (T19/05/14N33) "Cuando uno ya ha dado sugerencias entonces más adelante hacía que la tutoría fluyera más porque ella tenía como unas pautas, entonces se autocorregía más fácil". Este incremento en el dominio puede obedecer a que las prácticas desarrolladas por el tutor, según lo perciben algunos tutorados, están dirigidas a cualificar el desempeño escritor de su tutorado, más que a mejorar su texto: (Nota del grupo de discusión) "pero también una cosa que veo que de pronto el tutor siempre intenta cuando está dando la tutoría, es que uno trate de hacer el ejercicio por uno mismo para que él no sea imprescindible; es algo que con la persona o las personas que me tocó siempre tratan de hacer, esperan que uno trate de construir las cosas para que uno aprenda a hacer ese tipo de redacción y mejore [...]".

Se resalta que, a partir de la tutoría, el tutorado ve en la escritura de textos futuros, una tarea de largo aliento e inherente a las actividades investigativas y profesionales: (Nota del grupo de discusión) "[...], entonces la tutora siempre nos habla: cuando escribas el artículo, así se hace, entonces como que uno ya va visualizando otras cosas que puede escribir a partir de ya. Uno no se va a limitar, acabó el proyecto y ya, hasta ahí llega, sino que uno ya se prepara para escribir otras cosas". Esto también indica que los beneficios de la tutoría no se limitan a la producción del texto objeto de reflexión durante la sesión, sino que los tutorados plantean que los aprendizajes construidos allí benefician la ejecución de futuras tareas de escritura: (Nota del grupo de discusión) "Por ejemplo, con otras materias nos ponen a hacer ensayos y eso y uno ya tiene en cuenta esas cosas que nos han dicho en la tutoría para poder escribir otras cosas [...]".

Continuando con los aportes académicos, los participantes perciben aportes de la tutoría en escritura a las prácticas de lectura, lo cual parece lógico en 
atención a la estrecha relación entre lectura y la escritura. Uno de los primeros elementos que aparecen en relación con la lectura, es la adopción de criterios para la selección de textos de calidad y acordes a sus necesidades. Al respecto, un tutor expresa: (T6/05/14N19) "[...] entonces ellos se fueron con varias ideas de lo que tenían que hacer, cómo deben ser las fuentes que consultan, ummm a manera muy rápida se les explicó que, por ejemplo, uno debe pulirse más en cuanto a la bibliografía [...]". A su vez, un tutorado dice al respecto: (Tt6/05/14N19) "Sí, claro, porque nos mostró los errores que teníamos tanto en las diapositivas como en lo consultado y además aprendimos cómo consultar una fuente más... pues más acertada".

La lectura en voz alta, usualmente usada por tutor y tutorado durante su encuentro, se reconoce como una estrategia para identificar los errores que deben corregirse: (Tt16/05/14N31) "... cuando, cuando la escuchábamos a ella (a la tutora leer en voz alta) podíamos si... de la parte de receptores (mientras escuchan lo que la tutora lee), decir qué no sonaba bien o le faltaba algo y desde la parte nuestra también (cuando el tutorado leía en voz alta), porque uno se da cuenta si uno va comprendiendo lo que se estaba leyendo".

Además, los tutorados perciben que la necesidad de tener que discutir con un tutor de otra disciplina los planteamientos hechos en el texto que se revisa, los obliga a leer de una manera más detenida los textos que consultan previamente para fundamentar el propio: (Tt16/05/14N31) "Pues ummm, nos hizo hacer una lectura comprensiva, sí, tanto a mi compañero y a mí y que sea entendible para nosotros y para ella; en base a eso, continuábamos párrafo a párrafo".

El último beneficio relativo a la lectura, percibido por los participantes, se refiere a la posibilidad de volverse un lector más crítico, tanto de los propios textos como de los de otros autores, lo cual constituye un aprendizaje que seguramente permanece en el tiempo:

Nota del grupo de discusión:

"pues yo pienso que ya en la construcción del análisis de los datos de nuestro trabajo ya teníamos en cuenta como que no, mira, pongamos este conector, suena más bonito, ehh suena más agradable para el lector, entonces sí nos ha servido bastante y no solo para mí, sino para de pronto hay personas que me dicen: ve, ¿esto estará bien?, y yo leo y ahh sí, puedes cambiar esto y uno ya hace como el rol del tutor, entonces a mí me ha pasado con compañeros, entonces mirá, le podés cambiar esto y ponerle esto. Entonces ipor qué? por todos los conocimientos que las tutoras de aquí nos han enseñado a nosotros y entonces ya uno tiene en cuenta todo ese tipo de cosas y se le puede colaborar a otras personas".

Mejorar la dinámica para trabajar en grupo cuando se debe entregar un trabajo escrito conjuntamente también se percibe como aporte. Los tutorados perciben la tutoría como un espacio que les posibilita articular los aportes de cada miembro mediante la evaluación conjunta del texto: (Nota del grupo de discusión) "... o sea, como siempre pasa: tú haces esto, yo hago esto, yo hago esto y lo unimos y ya, pero no leemos lo que hizo mi compañera entonces en ese momento cuando estamos todos reunidos con la tutora es que ahí entendemos: no, mira que aquí falta esto, iah!, yo no sabía lo que era eso, o sea, son cosas que también... sí ayudan bastante para entender sobre el tema".

Finalmente, en relación con los aportes académicos, el aprendizaje del tema abordado en el texto que se revisa aparece como un beneficio. Un tutorado señala que "además es como que uno escribe pero uno se limita, 
porque uno no tiene todo el tiempo de leer y releer y releer, entonces uno llega acá: es un espacio muy bueno en el que uno se sienta, lee y uno dice qué duro pues, muy bueno pero no tiene nada qué ver con el tema y cosas así; entonces sí, como que uno dice ahhhh, me falta describir eso o sería bueno ponerle esto, [...] entonces uno aprende sobre el tema que está escribiendo" (Nota del grupo de discusión).

La posibilidad de potenciar la función epistémica de la escritura es percibida exclusivamente por los tutorados que cursan un programa académico diferente al del tutor, pues sienten la necesidad de aclarar para sí mismos las ideas que plantean, al tiempo que intentan explicarlas a alguien ajeno a su campo del saber. Quienes cursan el mismo programa académico, por su parte, perciben como beneficio que los tutores conozcan las demandas y estilos particulares de sus docentes y los orienten en ese sentido.

Percepciones sobre los aportes de las tutorías en el ámbito social

Los beneficios sociales percibidos por los participantes parecen ir más allá del espacio tutorial; por ejemplo, consideran que las tutorías les permiten ampliar su círculo de amistades: (Tt/25/04/14N3) "mmm, aparte de la escritura, como mejorar las relaciones con los estudiantes de otros programas, de otros semestres" y (T/27/04/14N4) "sí, de todos los tipos, social, he ampliado el círculo social, he aumentado el número de amigos en face ${ }^{2}<$ riéndose $>$ ".

También se reconocen como beneficios sociales aquellos que tienen qué ver con lo que impacta positivamente su desempeño en este rol: (T/08/04/14N8) “Eeeh..., bueno, en cuanto al tipo personal, [...] en mi primera tutoría, yo tenía mucho miedo <riéndose> de enfrentarme a un tutorado, en serio, ahora no, ahora es como si los conociera de toda la vida, sin llegar a ser confianzuda, por ejemplo: ihola, ¿cómo estás, Vane? iMucho gusto! Mira tal cosa, vamos a trabajar esto, igual siempre respetando el espacio de él como autor del texto, entonces en lo personal ha mejorado eso". En este registro, se observa que para el tutor es una ganancia adquirir confianza en sí mismo para ejercer su rol, el que además se aprende gracias a la práctica y en la medida en que van adquiriendo nuevos conocimientos en lectura y escritura académica, aspecto que no solo se refleja en las tutorías sino en las percepciones que tienen los tutorados de ellos.

\section{Percepciones sobre las características de los participantes}

Aquí se presentan las maneras como cada participante percibe al otro, teniendo en cuenta las actitudes, características y comportamientos que facilitan u obstaculizan el desarrollo de la tutoría. Se incluyen, además de las percepciones del tutor sobre el tutorado y viceversa, las que ha construido alguno de ellos respecto a otro tutor, a otro tutorado o sobre otros actores que participan activamente en el Centro de Escritura, aunque no en la tutoría, por ejemplo, su coordinadora.

Los tutores refieren valorar en los tutorados aspectos personales como el respeto: (T/08/04/14N8) "No, superreceptivo, superrespetuoso [...]", y otros referidos al papel de estos como aprendices, entre los que incluyen la participación activa, la capacidad de aprendizaje y la aceptación de sugerencias: (T/04/04/14N5) "eeeh..., pues es un chico con el que se trabaja muy chévere, es muy atento a las sugerencias que uno le da, eeeh... se ve que ha avanzado mucho pues con la tutoría de antes, pues se ve que ha aprendido bastante [...]".

2 Se refiere a Facebook, una red social virtual usada frecuentemente por los jóvenes. 
Sin embargo, los tutores también perciben en los tutorados duda y desconfianza: (T/25/03/14N2) "eeeh... la actitud era de duda, no tenía confianza en lo que de pronto... en las sugerencias que uno le hacía [...]", así como distracción y rechazo: (T6/05/14N19) "[...] sin embargo, había dos personas que no les importaba en lo más mínimo lo que tú estuvieras hablando y con la actitud como que todo te lo rechazaban [...]", y participaciones poco propositivas: (T/25/03/14N2) "[...] y entonces se quedaba por ejemplo mirando el computador y me miraba y no tenía una participación activa, sino que esperaba que yo le hiciera el trabajo y obviamente eso es un error"; aspectos que, según consideran, no favorecían el desarrollo de las tutorías.

A su vez, los tutorados también perciben en los tutores características personales como la amabilidad y la energía, así como características que serían atribuibles a su rol como tutor, tales como saber explicar, dominar el tema y aceptar el error como oportunidad de aprendizaje: (Tt6/05/14N19) "Es una chica muy amable, eeeh... nos supo explicar, tiene paciencia y además hizo que entráramos en confianza con ella o sea si nos equivocábamos, si no sabíamos, pues no pasaba nada, la idea es aprender". Es importante mencionar que los tutorados no manifestaron inconformidad, ni percibieron actitudes negativas por parte del tutor durante el desarrollo de la tutoría.

Por último, se encontró que los tutorados valoran la presencia de la coordinadora del Centro durante la ejecución de las sesiones, pues consideran que a pesar de su posición de docente, favorece la creación de un ambiente agradable y propicio para el trabajo a realizar, y ofrece respaldo a la labor de los tutores: (Nota del grupo de discusión) "y las tutoras van y le preguntan y ella (la coordinadora) está como abierta a todas las dudas, porque el tutor no puede resolver entonces le preguntan a ella y da también sus opiniones y soluciona las dudas".

\section{Percepciones sobre la relación entre pares}

Otro aspecto a resaltar es la percepción que surge sobre la interacción entre pares. El sentimiento de estar en una relación en la que no hay posiciones de autoridad facilita plantear las inquietudes y genera más confianza que cuando se interactúa con un docente, lo que se revela en los siguientes registros: (Tt16/05/14N31) "Me sentí muy bien porque hay como mayor confianza, que a veces uno con el profesor no tiene tanta confianza para hacerle muchas preguntas, pero con un estudiante es mucho más fácil" y (T/25/03/14N2) "pues me sentí, creo que me sentí más cómoda, sabiendo que ella es estudiante a que como la hubiera recibido de pronto con una profesora o algo, se siente más como chévere".
Sin embargo, es claro que los tutorados no solo valoran que se trate de estudiantes como ellos, sino que resaltan en los tutores su alto nivel de conocimientos en escritura: (Tt/04/04/14N5) "[...] me parece que para hacer esta clase de tutorías pues la persona debe tener gran capacidad y conocimiento para hacer esto y entonces pues sí, la niña (tutora) tenía estos aspectos [...]" y que cursan semestres superiores: (Tt/08/04/14N7) "[...] bien, porque igual ya son de semestres avanzados y ya ellos tienen como una capacitación que a uno lo pueden ayudar"; como garantía de éxito de la tutoría, lo cual relativiza la condición de par del tutor.

Resulta llamativo que, al comparar el tutor de escritura con el profesor del campo disciplinar, director de proyecto de investigación, en este caso, encuentren ventajas en el primero, debido a que no se limita a decir qué debe hacerse, sino que explica cómo hacerlo: "el tutor (director de tesis), el ingeniero, está para eso: para que nosotros sigamos las pautas y que todo lo que uno escribe se entienda, pero ciertamente él no nos ha podido aportar. Él nos lo dice: ustedes tienen que hacerlo de manera que todo el mundo lo entienda, ustedes tienen que entender que esto va a reposar en la biblioteca y que cualquier persona lo va a necesitar, y que cuando lo lea y lo vea esa persona va a tener que entenderlo; pero nos dice eso, pero no nos dice cómo hacerlo, pero en cambio acá (en el Centro de Escritura) nos dicen cómo hacer eso" (Nota del grupo de discusión).

Sin embargo, se encontró también que algunos tutorados asumen como su responsabilidad buscar ayuda para la escritura antes de enviar avances a su docente, de modo que este pueda invertir su tiempo en asuntos que ellos consideran más propios de su rol: "... en el caso nuestro, nosotros sí le avisamos al profe que vinimos (al Centro de Escritura), que le dijera a las personas porque es que uno sufre con eso, y yo digo: uno sufre haciéndolo y ellos (los profesores) sufren y pierden mucho tiempo, el profesor revisando algo que no está bien; entonces para que nos economicemos tiempo y ellos reciban algo bien escrito [...]" (Nota del grupo de discusión).

\section{Discusión}

Los hallazgos de este trabajo coinciden con los de otros que han revelado valoraciones positivas del trabajo entre pares, bien sea en torno a la escritura académica, en otros campos del saber o en la facilitación de la adaptación al ambiente universitario. Por ejemplo, concuerda con Gloria Mercedes Alzate-Medina y Luis Bernardo Peña-Borrero (2010), Claudia Esperanza Cardozo-Ortiz (2011), Jeffrey Lee Lucas (2000) y Lidón Moliner-Miravet, Auxiliadora Sales-Ciges y Odet 
Moliner-García (2014), Armandina Serna-Rodríguez y Griselda Cruces y Rojas (2010), quienes señalan que la relación entre pares no solo aporta a la solución de dificultades académicas, sino que contribuye al desarrollo de habilidades personales y sociales.

Entre los beneficios específicos sobre la escritura, los resultados de esta investigación concuerdan con otros según los cuales la tutoría entre iguales les permite a los estudiantes reconocer y diferenciar las características de los escritos académicos propios de la disciplina (Alzate-Medina \& Peña-Borrero, 2010). Así mismo, coinciden con Karen Shirley López (2012) y Alfonso Vargas-Franco (2014) en que el trabajo entre pares posibilita conocer el proceso para la construcción de un texto académico, reconocer la necesidad de ajustarse a la audiencia y la importancia de la validez de la información y las fuentes consultadas. Además, ayuda a corregir aspectos formales de la escritura, gracias al proceso de reflexión metalingüística suscitado durante la revisión, como encontraron Keith J. Topping, Rayenne Dehkinet, Sylvia Blanch-Gelabert, Mariona Corcelles-Seuba y David Duran-Gisbert (2013), así como al conocimiento de las normas de presentación de trabajos y al uso de fuentes bibliográficas que los tutores aportaron, como arrojó el estudio de Clemente Lobato, Felisa Arbizu y Laura del Castillo (2004).

Estudios previos no han referido otros beneficios específicos que trae la tutoría de escritura académica entre pares sobre la lectura del tutorado, como la exigencia de leer de manera más atenta un documento que consulta para poder explicar sus planteamientos centrales a un tutor que no comparte su campo disciplinar. Sin embargo, resultan interesantes al respecto los hallazgos de Keith J. Topping, Rayenne Dehkinet, Sylvia Blanch-Gelabert, Mariona Corcelles-Seuba y David Duran-Gisbert (2013), según los cuales algunos textos llevados por los tutorados contienen tantos errores que suponen un mayor reto para el tutor, quien debe identificar en ellos las ideas básicas y conectarlas con el fin de atribuirles significado. Ambos resultados revelan el esfuerzo que suponen las actuaciones de cada participante por leer detenidamente los documentos pensando en el bienestar del otro. Tampoco se encontraron en reportes de otras investigaciones, aportes de la tutoría entre pares a la dinámica de trabajo en grupo orientado a la producción de un texto, asunto revelado en este estudio.

Sobre las percepciones de los participantes con respecto a que las tutorías sean desarrolladas por pares, tutores y tutorados coincidieron en resaltar un ambiente de confianza y calidez, donde hay menos tensiones que las propias de la relación entre docente y estudiante, como encontraron Claudia Esperanza Cardozo-Ortiz (2011), Lidón Moliner-Miravet, Auxiliadora Sales-Ciges y Odet Moliner-García (2014) y Keith
J. Topping (2015). Además, así como señalan los estudios de Jolan T. Nisbet, Mark D. Haw y Ashleigh J. Fletcher (2014), los tutores participantes en esta investigación manifiestan que la interacción con sus tutorados les permite ir adquiriendo confianza sobre sus conocimientos y sobre su papel como tutor para desempeñar mejor su trabajo.

Por eso, resulta comprensible que los tutorados destaquen en el tutor cualidades como la amabilidad, cercanía, calidez y respeto, aunque también son altamente valoradas condiciones como los conocimientos en escritura y la capacidad para orientar. Así lo identificaron Pedro Ricardo Álvarez-Pérez y Miriam Catalina González-Afonso (2005) cuando, al pedir a los estudiantes enumerar las capacidades que debía poseer un compañero tutor, señalaron algunas personales como empatía, al tiempo que resaltaron la importancia de que conocieran la información necesaria para desarrollar su labor. Es claro, entonces, que los tutorados valoran tanto los rasgos propios de una relación entre pares, como aquellos propios de su rol de tutores; la combinación entre amable como par y confiable como tutor resulta altamente apreciada por los tutorados.

De igual manera, los tutores valoran en los tutorados aquellos rasgos atribuibles a los buenos aprendices, como la capacidad de acoger sugerencias y la participación activa, cualidades que, de acuerdo con Luis Enrique Jiménez-Becerril (2009), son deseables en ese rol y según Madai Silva-González y Margarita Cárdenas-Jiménez (2008) generan aprendizajes tanto en el tutor como en el tutorado. En coherencia, los tutores identificaron la duda, desconfianza o actitud pasiva como actitudes de los tutorados que no contribuyen a los propósitos de la tutoría. Tales actitudes podrían explicarse por las motivaciones que llevan a algunos tutorados a asistir al Centro de Escritura: cumplir una exigencia del docente, lo que les impide asumirlo como una posibilidad de aprendizaje a aprovechar. Sin embargo, Lidón Moliner-Miravet, Auxiliadora Sales-Ciges y Odet Moliner-García (2014) refieren que los estudiantes, a medida que avanzaban con sus tutorías, se hacían más responsables con sus labores académicas, además de sentirse más autónomos e independientes, lo que podría explorarse en el Centro de escritura como objeto de estudio, más adelante.

Se encontró en este estudio que algunos tutorados, antes de la sesión de tutoría, sentían desconfianza sobre las competencias de los tutores para apoyarlos, por ser estudiantes universitarios como ellos. Tal desconfianza desapareció durante la sesión al evidenciar altas competencias en los tutores, lo que no ocurrió en los trabajos de Karen Shirley López (2012) y Alfonso Vargas-Franco (2014), en los que algunos participantes reportaron, al final de las secuencias de enseñanza y aprendizaje, que los estudiantes no tienen los saberes 
necesarios para revisar los textos de sus compañeros, labor que consideran debe ser asumida por un profesor. Sin embargo, es clara la diferencia entre los contextos de indagación de esos trabajos y aquel en el que se llevó a cabo esta investigación. Los primeros estuvieron constituidos por aulas de clase en las que todos los participantes cumplen el rol de estudiantes y los papeles de revisor y autor son desarrollados por cada uno a solicitud de un profesor que evaluará y calificará el desempeño de todos. El segundo, en cambio, fue un Centro de Escritura al que asisten estudiantes de diferentes carreras y semestres con el propósito de recibir apoyo para revisar su texto; el rol de tutor es asumido por universitarios seleccionados y formados de manera continua; no es un rol alternado con los tutorados.

En las secuencias didácticas se trata claramente de una relación entre iguales, simétrica, que Karen Shirley López (2012) y Alfonso Vargas-Franco (2014) denominan revisión entre iguales y revisión entre pares, respectivamente. Esto no es exactamente lo que sucede en el Centro de Escritura de la Universidad del Cauca: allí los asistentes esperan encontrar a alguien con mayores competencias escriturales y capacidad de orientación; en esta condición se llevan a cabo tutorías entre pares. Estudios como el de Gloria Mercedes Alzate-Medina y Luis Bernardo Peña-Borrero (2010) también han documentado tutorías entre pares en las que quienes fungen como tutores cursan semestres más avanzados y no alternan su rol con los tutorados, contrario a la mirada de Rosmar Guerrero y Rudy Mostacero (2014), quienes hablan de la tutoría grupal entre iguales, caracterizada por la simetría y los roles intercambiables, en un grupo de escritura de posgrado.

A continuación, se plantean algunas conclusiones que pueden derivarse de los hallazgos de este estudio.

\section{Conclusiones}

Los hallazgos de esta investigación revelan que tutor y tutorado perciben beneficios, tanto en el campo académico como en el social, producto de su participación en las tutorías de escritura académica en el Centro de Escritura de la Universidad del Cauca. Esto permite inferir que la tutoría entre pares, asumida como una relación parcialmente asimétrica, puede ser una estrategia valiosa en los centros de escritura para favorecer el aprendizaje de la producción textual, si se cuenta con condiciones como la formación permanente de tutores.

Sin embargo, esta investigación no estuvo orientada a evaluar el desarrollo escritural o académico como producto de las tutorías. Para ello, se requerirán otras investigaciones — podrían ser causales — que no se basen exclusivamente en las percepciones de los participantes y puedan dar cuenta del impacto de las acciones desarrolladas. También serían deseables trabajos que exploren las percepciones de profesores y directivos sobre las tutorías de escritura académica desarrolladas en el Centro de Escritura, para tener acceso a las apreciaciones de todos los actores involucrados.

Conviene señalar como limitaciones de este trabajo la imposibilidad de generalizar sus hallazgos, debido al tamaño de la población sujeto de estudio, aunque dada su naturaleza, tampoco era su objetivo. En ese sentido, los resultados son aplicables para el espacio investigado, aunque también pueden servir como referencia a otros centros y programas de escritura, para la reflexión sobre las prácticas que allí llevan a cabo.

Para terminar, conviene decir que los hallazgos de esta investigación permitieron al equipo de trabajo del centro de escritura analizado discutir posibles acciones para cualificar sus servicios. Por ejemplo, se analizaron maneras de potenciar la función epistémica de la escritura en estudiantes 
que cursan la misma carrera que los tutores, pues solo los tutorados de otros programas académicos percibieron ese beneficio. Dar a conocer los hallazgos de esta investigación también ha visibilizado más el centro de escritura, lo cual ha incrementado el número de asistentes para el servicio de tutorías. Realizar este tipo de estudios en los espacios educativos resulta necesario para documentar las maneras en que los beneficiarios perciben los servicios que se les ofrecen, de modo que los equipos oferentes puedan retroalimentarse y tomar decisiones respecto a sus cursos de acción.

\section{Agradecimientos}

Participaron en la recolección y análisis de información las entonces estudiantes de fonoaudiología Stefany Figueroa, Catherine González, Yessica Pino y Tania Rendón. Resultados preliminares de este trabajo fueron socializados en el Primer Encuentro Internacional de Lectura y Escritura en las disciplinas de la Educación Media y Superior, Cuenca, Ecuador, en enero de 2015; en el II Congreso Internacional: Lectura y Escritura en la Sociedad Global, Barranquilla, Colombia en junio de 2015 y en el IV Seminario Internacional de Lectura en la Universidad, III Congreso Nacional de Expresiones de Cultura Escrita en Instituciones de Educación Media Superior y Superior, y $\vee$ Seminario Internacional de Cultura Escrita y Actores Sociales, en Tlaxcala, México, octubre de 2015.

\section{Sobre los autores}

Pilar Mirely Chois-Lenis es fonoaudióloga. Especialista en la enseñanza de la lectura y la escritura en lengua materna. Magíster en lingüística y español. Estudiante de doctorado en ciencias de la educación. Ha investigado y publicado sobre la enseñanza de la lectura y la escritura en grados iniciales de escolaridad y en el ámbito universitario.

Adriana Carolina Casas-Bustillo es fonoaudióloga. Especialista en pedagogía de la lectura y la escritura. Estudiante de maestría en psicología con énfasis en cognición y lenguaje. Docente universitaria con intereses particulares en investigación y formación académica en lectura y escritura en todos los niveles académicos.

Amparo López-Higuera es fonoaudióloga. Especialista en pedagogía y magíster en desarrollo infantil. Docente del programa de fonoaudiología. Estudiante del doctorado en ciencias sociales, niñez y juventud. Ha investigado en las líneas de educación y currículo y lenguaje.

Diana Marcela Prado-Mosquera es fonoaudióloga. Estudiante de la maestría en educación: desarrollo humano. Docente del programa de fonoaudiología. Coordinadora del Centro de Escritura, Universidad del Cauca, Sede Norte. Ha investigado en las líneas de lectura y escritura académica.

Edwin Yamir Cajas-Paz es fonoaudiólogo. Estudiante: especialización en audiología. Se desempeña actualmente como fonoaudiólogo en el área de salud ocupacional y docente universitario, en programas de psicología, sobre percepción y desarrollo auditivo. Sus intereses por la investigación se direccionan hacia su profesión.

\section{Referencias}

Adams, Howard G. (1992). Mentoring: An Essential Factor in the Doctoral Process for Minority Students. (ERIC Document Reproduction Service No. ED 358 769.) Disponible en: http://eric.ed.gov/?id=ED358769 
Álvarez-Pérez, Pedro Ricardo \& González-Afonso, Miriam Catalina (2005). La tutoría entre iguales y la orientación universitaria: una experiencia de formación académica y profesional. Educar, 36, 107-128. Disponible en: https://ddd.uab.cat/pub/educar/0211819Xn36/0211819Xn3 6p107.pdf

Alzate-Medina, Gloria Mercedes \& Peña-Borrero, Luis Bernardo (2010). La tutoría entre iguales: una modalidad para el desarrollo de la escritura en la educación superior. Universitas Psychologica, 9 (1), 123-138. Disponible en; http://revistas.javeriana.edu.co/index.php/revPsycho/ article/view/717/428

Asociación Nacional de Universidades e Instituciones de Educación Superior, ANUIES (2000). Programas institucionales de tutoría: una propuesta para la organización y funcionamiento en las instituciones de educación superior. Disponible en: http://evirtual.uas/p.mx/FCQ/ tutorias/Documentos\%20compartidos/INTRODUCCION/PROGRA MAS\%20INSTITUCIONALES\%20DE\%20TUTORIA_ANUIES.pdf

Boyle, Joyceen S. (2003). Estilos de etnografía. En Janice M. Morse \& Joan L. Bottorff (eds.). Asuntos críticos en los métodos de investigación cualitativa, 185-214. Medellín: Editorial Universidad de Antioquia.

Cardozo-Ortiz, Claudia Esperanza (2011). Tutoría entre pares como una estrategia pedagógica universitaria. Educación y Educadores, 14 (2), 309-325. Disponible en: http://www.scielo.org.co/pdf/eded/v14n2/ v14n2a05.pdf

Carlino, Paula (2008a). Desafíos para hacer una tesis de posgrado y dispositivos institucionales que favorecerían su completamiento. Instituto de Lingüística de la Universidad de Buenos Aires. Conferencia plenaria en el Segundo Encuentro Nacional y Primero Internacional sobre Lectura y Escritura en Educación Superior, organizado por la Red Nacional para el Desarrollo de la Lectura y Escritura en la Educación Superior (REDLEES), la Asociación Colombiana de Universidades (ASCUN) y la Pontificia Universidad Javeriana de Bogotá, Bogotá, 18 y 19 de septiembre de 2008. Disponible en: http://www.aacademica. org/paula.carlino/132.pdf

Carlino, Paula (2008b). Revisión entre pares en la formación de posgrado. Lectura y Vida: Revista Latinoamericana de Lectura, 29 (2), 20-28. Disponible en: http://www.lecturayvida.fahce.unlp.edu.ar/numeros/ a29n2/29_02_Carlino.pdf

Carlino, Paula (2009). Exploración de géneros, diario de tesis y revisión entre pares: análisis de un ciclo de investigación-acción en talleres de tesis de posgrado. En Elvira Narvaja de Arnoux (ed.). Escritura y producción de conocimiento en las carreras de posgrado, 220-239. Buenos Aires: Santiago Arcos. Disponible en: http://www.aacademi ca.org/paula.carlino/182.pdf

Cassany, Daniel (1999). Construir la escritura. Barcelona: Paidós.

Castelló, Montserrat; González, Dolores \& Iñesta, Ana (2010). La regulación de la escritura académica en el doctorado: el impacto de la revisión colaborativa en los textos. Revista Española de Pedagogía, 68 (247), 521-537. Disponible en: https://www.jstor.org/ stable/23766358?seq=1\#page_scan_tab_contents

Colvin, Janet W. \& Ashman, Marinda (2010). Roles, Risks, and Benefits of Peer Mentoring Relationships in Higher Education. Mentoring \& Tutoring: Partnership in Learning, 18 (2), 121-134. doi: 10.1080/13611261003678879. Disponible en: http://www.wsac. wa.gov/sites/default/files/2014.ptw.(37).pdf 
Corcelles-Seuba, Mariona; Cano-Ortiz, Maribel; Bañales-Faz, Gerardo \& Vega, Norma Alicia (2013). Enseñar a escribir textos científico-académicos mediante la revisión colaborativa: El trabajo final de grado en Psicología. REDU, Revista de Docencia Universitaria, 11 (1), 79-104. Disponible en: http://polipapers.upv.es/index.php/REDU/article/ view/5593/5584

Domingo, Joan (2008). El aprendizaje cooperativo. Cuadernos de Trabajo Social, 21, 231-246. Disponible en: https://revistas.ucm.es/index.php/CUTS/ article/download/CUTS0808110231A/7531

Duque-Ossa, Lina Marcela; Gómez-Martínez, Vittoria \& Gaviria-Cano, Ana Sofía (2009). Tutorías académicas pares. Pereira: Colombia Aprende, Universidad Católica Popular del Risaralda. Disponible en: http://www.colombiaaprende.edu. co/html/micrositios/1752/articles-323200_recur so_2.pdf

Dysthe, Olga \& Lillejord, Sølve (2012). From Humboldt to Bologna: Using Peer Feedback to Foster Productive Writing Practices among Online Master Students. International Journal of Web Based Communities, 8 (4), 471-485. doi:10.1504/ IJWBC. 2012.049561

Ehrich, Lisa C.; Hansford, Brian C. \& Tennent, Lee (1113 de septiembre de 2003). Mentoring in Medical Context. Conferencia llevada a cabo en la British Educational Research Association Annual Conference, Heriot Watt University, Edinburgh. Disponible en: http://eprints.qut.edu.au/2894/1/ Medical_Contexts_and_Mentoring_4.pdf

Galbraith, Jonathan \& Winterbottom, Mark (2011). Peer Tutoring: What's in it for the Tutor? Educational Studies, 37 (3), 321-332.

Glaser, Barney G. \& Strauss, Anselm L. (1967). The Discovery of Grounded Theory: Strategies for Qualitative Research. Chicago: Aldine Publishing Company.

Govea-Rodríguez, Violeta Margarita; Vera, George \& Vargas, Aura Marina (2011). Etnografía: una mirada desde corpus teórico de la investigación cualitativa. Omnia, 17 (2), 26-39. Disponible en: http://200.74.222.178/index.php/omnia/article/ view/7355/7344

Grupo de Investigación sobre Aprendizaje entre Iguales, GRAl, de la Universidad Autónoma de Barcelona (2007). Tutoría entre iguales. Universidad Autónoma de Barcelona. Disponible en: http:// grupsderecerca.uab.cat/grai/es, http://grupsde recerca.uab.cat/grai/es/content/tutoria-entreiguales

Guardiola-Jiménez, Plácido (2013). La percepción. Universidad de Murcia.
Guerrero, Rosmar \& Mostacero, Rudy (2014). Logros y alcances de escribir el trabajo de grado en un grupo de escritura. Investigación Arbitrada, 18 (61), 527-534. Disponible en: http://www.saber. ula.ve/bitstream/123456789/39769/1/art12.pdf

Jaramillo-Echeverri, Luis Guillermo \& Aguirre-García, Juan Carlos (2012). La percepción y la pregunta por el sentido: implicaciones para una enseñanza corporal con-sentido. Lúdica Pedagógica, 2 (17), 33-40. Disponible en: http://revistas.pe dagogica.edu.co/index.php/LP/article/viewFi le/1774/1752

Jiménez-Becerril, Luis Enrique (2009). La actitud del tutor y tutorado ante el programa institucional de tutorías en la Universidad Pedagógica Nacional Unidad-Ajusco (Tesis de Grado). Universidad Pedagógica Nacional, Unidad-Ajusco, Ajusco, México.

Johnson, David W.; Johnson, Roger T. \& Holubec, Edythe J. (1999). El aprendizaje cooperativo en el aula. Buenos Aires: Paidós.

Josgrilberg, Fabio Botelho (2008). A fenomenologia de Maurice Merleau-Ponty e a pesquisa emcomunicaçâo. Revista Fronteiras, 8 (3), 223-232. Disponible en: http://revistas.javeriana.edu.co/ index.php/signoypensamiento/article/viewFi le/4579/3549

Lobato, Clemente; Arbizu, Felisa \& Del Castillo, Laura (2004). Las representaciones de la tutoría universitaria en profesores y estudiantes: estudio de un caso. Educación XX1, 7 (1), 135-168. Disponible en: http://revistas.uned.es/index.php/educa cionXX1/article/view/333/289

Loke, Alice J. T. Yuen \& Chow, Filomena L. W. (2007). Learning Partnership -The Experience of Peer Tutoring among Nursing Students: A Qualitative Study. International Journal of Nursing Studies, 44 (2), 237-244. Disponible en: https://www. academia.edu/19868059/Learning_partners hip_the_experience_of_peer_tutoring_among_ nursing_students_A_qualitative_study

López, Karen Shirley (2012). La revisión entre pares de tareas de escritura como herramienta de una didáctica metacognitiva en el aula de lengua. Lenguaje, 40 (2), 351-381. Disponible en: http:// revistalenguaje.univalle.edu.co/index.php/Len guaje/article/view/1735/1678

Lucas, Jeffrey Lee (2000). Mentoring as a Manifestation of Generativity among University Faculty. (Thesis of PhD). George Fox University, Oregon, United States.

Lyons, William; Scroggins, Don \& Rule, Patra Bonham (1990). The Mentor in Graduate Education. Studies in Higher Education, 15 (3), 277-285. 
Merleau-Ponty, Maurice (1975). Fenomenología de la percepción. Barcelona: Península.

Moliner-Miravet, Lidón; Sales-Ciges, Auxiliadora \& Moliner-García, Odet (2014). An Experience of Reciprocal Peer Tutoring at the University. Procedia - Social and Behavioral Sciences, 116, 2809-2812.

Mostacero, Rudy (2014). La tutoría grupal y entre iguales en el contexto de los estudios de posgrado. Legenda, 18 (18), 67-97. Disponible en: http://erevistas.saber.ula.ve/index.php/legenda/article/view/5207/5003

Moust, Jos H. C. \& Schmidt, Henk G. (1994). Effects of Staff and Student Tutors on Student Achievement. Higher Education, 28 (4), 471-482.

Nisbet, Jolan T.; Haw, Mark D. \& Fletcher, Ashleigh J. (2014). The Role of Tutors in Peer Led Teaching. Education for Chemical Engineers, 9 (1), 15-19.

Panitz, Theodore (1999). Collaborative versus Cooperative Learning: A Comparison of the Two Concepts Which Will Help Us Understand the Underlying Nature of Interactive Learning. (ERIC Document Reproduction Service No. ED 448 443). Disponible en: http://files.eric. ed.gov/fulltext/ED448443.pdf

Pardo, Marta \& Castelló, Monserrat (2016). Teaching Writing for Learning at University: a Proposal Based on Collaborative Review / Enseñar a escribir para aprender en la universidad: una propuesta basada en la revisión colaborativa. Infancia y Aprendizaje, 39 (3), 560-591. doi: 10.1080/02103702.2016.1196914

Pereira, Cecilia \& Stefano, Mariana di (2007). El taller de escritura en posgrado: representaciones sociales e interacción entre pares. Revista Signos, 40 (64), 405-430. Disponible en: http://www.scielo.cl/scielo. php?script=sci_arttext\&pid=S0718-09342007000200007

Roscoe, Rod D. \& Chi, Michelene T. H. (2008). Tutor Learning: the Role of Explaining and Responding to Questions. Instructional Science, 36, 321-350. Disponible en: http://chilab.asu.edu/papers/roscoe\%20 chi\%20tutor\%20learning\%202008.pdf

Santiviago, Carina \& Mosca, Aldo (2012). Fundamentos conceptuales de las tutorías entre pares. La experiencia de la Universidad de la República. Montevideo. Disponible en: http://data.cse.edu.uy/sites/data.cse. edu.uy/files/diagramacion_TEP_I_corregido4.pdf

Serna-Rodríguez, Armandina \& Cruces y Rojas, Griselda (2010). La tutoría académica desde la perspectiva de los alumnos. Conferencia llevada a cabo en el Primer Encuentro Nacional de Tutoría Colima, Colima, México. Disponible en: http://148.213.1.36/Documentos/Encuentro/ PDF/7.pdf

Sharif, Nurhidayah Mohd; Zakaria, Mohamad Hassan; Wan Mansor, Wan Fara Adlina; Nordin, Norul Alima; Ng, Siew Fong \& Mustafa, Hema Rosheny (2012). Peer-tutoring and Tertiary ESL Learners. Procedia Social and Behavioral Sciences, 66, 441-447. Disponible en: http:// www.sciencedirect.com.acceso.unicauca.edu.co/science/article/pii/ S1877042812052731

Silva-González, Madai \& Cárdenas-Jiménez, Margarita (2008). Percepción de los coordinadores, tutores y alumnos en relación a la tutoría como estrategia de enseñanza aprendizaje. Enfermería Universitaria, 5 (3), 37-41. http://revistas.unam.mx/index.php/reu/article/view/30163

Sinner, Carsten (2001). Corpus oral de profesionales de la lengua castellana en Barcelona. Universidad Leipzig, Alemania.

Topping, Keith J. (1996). The Effectiveness of Peer Tutoring in Further and Higher Education: A Typology and Review of the Literature. Higher Education, 32 (3), 321-345. doi: 10.1007/BF00138870 
Topping, Keith J. (2015). Peer Tutoring: Old Method, New Developments / Tutoría entre iguales: método antiguo, nuevos avances. Infancia y Aprendizaje, 38 (1), 1-29. doi: 10.1080/02103702.2014.996407

Topping, Keith J.; Dehkinet, Rayenne; Blanch-Gelabert, Sylvia; CorcellesSeuba, Mariona \& Duran-Gisbert, David (2013). Paradoxical Effects of Feedback in International Online Reciprocal Peer Tutoring. Computers \& Education, 61, 225-231. doi: 10.1016/j.compedu.2012.10.002

Valles, Miguel S. (1999). Técnicas cualitativas de investigación social: reflexión metodológica y práctica profesional. Madrid: Síntesis.

Vargas-Franco, Alfonso (2013). Revisión entre iguales, escritura académica e identidad en la formación docente en la universidad colombiana (Tesis de Doctorado). Universitat Pompeu Fabra, Barcelona, Cataluña. Recuperado de http://www.tdx.cat/bitstream/handle/10803/125115/ tavf.pdf?sequence $=1$

Vargas-Franco, Alfonso (2014). Revisión entre iguales y escritura académica en la universidad: la perspectiva del estudiante. Folios: Revista de la Facultad de Humanidades, 39, 13-29. Disponible en: http://www. scielo.org.co/pdf/folios/n39/n39a02.pdf

Vargas-Jiménez, Ileana (2012). La entrevista en la investigación cualitativa: nuevas tendencias y retos. Calidad en la Educación Superior, 3 (1), 119-139. Disponible en: http://investiga.uned.ac.cr/revistas/index. php/revistacalidad/article/view/436/331

Venables, Anne \& Summit, Raymond (2003). Enhancing Scientific Essay Writing Using Peer Assessment. Innovations in Education and Teaching International, IETI, 40, 281-290. doi:10.1080/14703290320 00103816. Disponible en: https://www.researchgate.net/publica tion/37376992_Enhancing_scientific_essay_writing_using_peer_as sessment 